\title{
Percepção e Processamento Visual da Forma em Humanos: Filtros de Freqüências Radiais de 1 e 4 cpg
}

\author{
Natanael Antonio dos Santos ${ }^{1}$ \\ Maria Lúcia de Bustamante Simas \\ Universidade Federal de Pernambuco
}

\begin{abstract}
Resumo
O objetivo deste trabalho foi mensurar curvas de resposta ao contraste para os filtros de freqüências radiais de banda estreita de 1 e 4 cpg para padrões espaciais modulados pela função cilíndrica de Bessel $\left(J_{0}\right)$. Foram estimadas 15 curvas para cada filtro com o paradigma psicofísico de somação de resposta de supra-limiar aliado ao procedimento da escolha forçada. Participaram deste experimento 5 voluntários adultos com acuidade visual normal ou corrigida. Os resultados demonstraram somações máximas de limiar de contraste na freqüência de teste dos filtros de 1 e 4 cpg circundadas por inibições nas freqüências vizinhas às freqüências radiais de teste de cada filtro. Estes resultados são consistentes com a existência de filtros de freqüências radiais de banda estreita operando no sistema visual humano através do processo de somação ou inibição em faixa de freqüências específicas.

Palavras-chave: Processamento visual de forma; filtro radial; freqüência radial; somação de supra-limiar.
\end{abstract}

\section{Perception and Visual Processing of Form in Humans: Radial Frequency Filters of 1 and 4 cpd}

\begin{abstract}
The aim of this work was to measure narrow-band frequency response curves for two radial frequency filters, spatial stimuli modulated by cylindrical Bessel profiles, $J_{0}$, whose test frequencies were 1 and $4 \mathrm{cpd}$. Five humans observers with normal or corrected visual acuity measured 15 curves for each filter with a supra-threshold response summation psychophysics method allied within a forced-choice paradigm. The results showed maximum summation effects at test frequency for filters 1 and $4 \mathrm{cpd}$ surrounded on both sides by strong inhibition. These results are consistent with the existence of narrow-band radial frequency filters operating in human visual system either through summation or inhibition of specific frequency ranges.

Keywords: Visual processing of form; radial frequency filter; radial frequency; supra-threshold summation method.
\end{abstract}

A primeira proposta de estudar padrões em coordenadas polares ao invés de coordenadas cartesianas (grade senoidal) partiu de Kelly (1960), que sugeriu estímulos circularmente simétricos, os quais ele denominou de alvo $\mathrm{J}_{0}$, isto é, estímulos modulados pela função cilíndrica de Bessel de ordem zero. Esta classe de padrões tem recebido outras denominações, como por exemplo, alvo olho de boi (Bull's-eye), grades circulares, dentre outras. Nós temos denominado de estímulo elementar de freqüência radial porque a modulação de contraste varia senoidalmente ou cossenoidalmente na direção radial, considerando o centro do círculo como a origem do sistema de coordenadas polares (Figura 1). Kelly discutiu que padrões deste tipo poderiam ser mais apropriados para estudar o sistema visual considerando a simetria aproximadamente circular da retina.

\footnotetext{
${ }^{1}$ Endereço para correspondência: Laboratório de Percepção Visual, Departamento de Psicologia, $9^{\circ}$ andar, Centro de Filosofia e Ciências Humanas, Universidade Federal de Pernanbuco, 50670-901, Recife,PE. Fone (81) 32713734, Fax (81) 32718271. E-mail: natanael@npd.ufpe.br
}

Apesar de Kelly ter proposto estímulo dessa natureza desde 1960, o mesmo só foi utilizado 15 anos depois, quando Kelly e Magnuski (1975) utilizaram padrões de freqüências radiais circularmente simétricos para estimar a função de sensibilidade ao contraste do sistema visual humano, SVH. De acordo com estes autores, padrões modulados pela função cilíndrica de Bessel parecem ser mais naturais como estímulo visual e fornecem um centro claro de fixação. O mesmo não acontece com padrões paralelos, por exemplo, grade senoidal (Figura 4). O principal objetivo de Kelly e Magnuski foi comparar a função de sensibilidade ao contraste estimada para grades senoidais com a função de sensibilidade ao contraste estimada para freqüências radiais moduladas pela função cilíndrica de Bessel, $\mathrm{J}_{0}$. O fato é que nenhum outro artigo aparece na literatura até 1982, quando Kelly replicou parte do estudo de 1975, com a técnica psicofísica de ajustamento de contraste, onde o voluntário ajustava seu próprio limiar de contraste. Os resultados obtidos por Kelly demonstraram que a faixa de maior sensibilidade para $\mathrm{J}_{0}$ foi por volta de 0,5-2,0 cpg. Os resultados de Kelly também mostraram que o SVH é mais sensível 
para estímulos do tipo grade senoidal do que para estímulos radiais.

Em 1996, Santos mediu a sensibilidade ao contraste para estímulos radiais modulados pela função esférica de Bessel para vários jn $(n=0,1,2,4,8$ e 16) com o paradigma psicofísico da escolha forçada. O $n$ representa a ordem da função de Bessel. Os resultados demonstraram que a faixa de máxima sensibilidade para estes estímulos depende da ordem da função. Por exemplo, a sensibilidade do SVH para $j_{0}$ se deu por volta de $1 \mathrm{cpg}$, enquanto que para $j_{16}$ se deu por volta de 2 e 3 cpg (Santos, 1996; Simas \& Santos 1997).

Em 1999, Santos também mediu a sensibilidade do SVH para estímulos modulados pela função cilíndrica de Bessel $\left(J_{0}\right)$ com o paradigma psicofísico da escolha forçada. Neste estudo, utilizou-se estímulos semelhantes ao de Kelly (Kelly, 1960, 1982; Kelly \& Magnuski, 1975). Os resultados encontrados foram semelhantes ao de Kelly, isto é, a sensibilidade máxima do SVH ocorreu em torno da freqüência radial de $1 \mathrm{cpg}$.

Atualmente, podemos encontrar na literatura outros trabalhos com estímulos concentricamente simétricos, por exemplo (Amidor, 1997; Gallant, Brau \& van Essen, 1993; Gallant, Connor, Rakshit, Lewis \& van Essen, 1996; Hegde \& van Essen, 2000; Hess, Wang, Demanins, Wilkinson \& Wilson, 1999; Verrall \& Kakarala, 1998; Wilkinson, Wilson \& Habak, 1998; Wilson \& Wilkinson, 1997, 1998; Wilson, Wilkinson \& Asaad, 1997), entretanto, estes utilizam sugestões teóricas diferentes de Kelly (Kelly, 1960; Kelly, 1982; Kelly \& Magnuski, 1975), Santos (1996, 1999) e Simas (Simas, 1985; Simas \& Dodwell, 1990; Simas \& Santos, 1997, 1998). Por exemplo, os estudos de Gallant e colaboradores (1993, 1996) utilizaram estímulos com modulação de onda senoidal ou quadrada baseados em freqüências radiais e angulares para testar a resposta de células na área visual V4 de macacos.

\section{Filtros de Freqüências Espaciais no Sistema Visual}

A idéia de canais múltiplos de freqüências espaciais foi proposta inicialmente por Campbell e Robson (1968). Essa abordagem assume que o SVH responde seletivamente a freqüências espaciais, ou seja, o sistema visual processa simultaneamente diferentes faixas estreitas de freqüências espaciais (Blakemore \& Campbell, 1969b; Campbell \& Robson, 1968; Sachs, Nachmias \& Robson, 1971). Em outras palavras, a abordagem de canais múltiplos ou análise de sistema linear defende que a percepção de um objeto (ou cena visual) qualquer ocorre porque o nosso sistema visual executa uma análise ou decomposição desse padrão complexo em seus componentes mais elementares, uma espécie de análise de Fourier. Santos e Simas (2001a) discutem boa parte das evidências psicofísicas e neurofisiológicas favoráveis a abordagem de canais múltiplos de freqüências espaciais.

Os estudos clássicos que procuraram caracterizar esses mecanismos responsáveis pelo processamento de contraste no SVH se basearam principalmente em resultados com grades senoidais verticais, estímulos semelhantes aos encontrados na Figura 4 (Blakemore \& Campbell, 1969a, 1969b; Blakemore, Nachmias \& Sutton, 1970; Campbell \& Maffei, 1970, 1974; Campbell \& Robson, 1968; Graham, 1972; Maffei \& Fiorentini, 1973; Pantle \& Sekuler, 1968). Nas últimas décadas, alguns pesquisadores procuraram estabelecer a existência de filtros ou canais para padrões de freqüências espaciais diferentes de grade senoidal, por exemplo, freqüências angulares (Santos, 1999; Simas, 1985; Simas \& Dodwell, 1990; Simas, Frutuoso \& Vieira, 1992; Simas \& Santos, 1998).

Do ponto de vista psicofísico um canal ou um filtro corresponde a uma estrutura visual independente que lida com uma faixa estreita de freqüência espacial (Santos, 1999; Santos \& Simas, 2001a).

Em 1985, Simas mensurou a resposta do SVH para o filtro de freqüência angular de 24 ciclos. Posteriormente, Simas e colaboradores estimaram a resposta do SVH para um número maior de filtros de freqüências angulares, como por exemplo os filtros angulares de 2, 4, 9, 13, 16, 24 e 47 ciclos (Simas \& Dodwell, 1990; Simas \& cols., 1992). Esses trabalhos foram realizados com o método psicofísico de somação de resposta de supra-limiar aliado ao procedimento da escolha forçada. Os resultados encontrados por Simas e colaboradores demonstraram efeitos de somação absoluta e relativa nas freqüências angulares de teste dos filtros circundadas por inibições. O que levou os autores a concluírem a favor da existência de alguma seletividade para faixas específicas de freqüências angulares.

\section{O Paradigma Psicofísico de Somação de Resposta de Supra-limiar}

O paradigma psicofísico de somação de resposta de surpa-limiar é uma adaptação do paradigma psicofísico de somação de resposta de sub-limiar de Kulikowski e King Smith (1973). No estudo original de Kulikowski e King Smith, eles empregaram $1 / 2$ do contraste necessário para alcançar o limiar de contraste dos respectivos estímulos de teste e de fundo (ruído). A finalidade foi obter, eventualmente, a somação do contraste da freqüência de teste com o contraste da freqüência de fundo e alcançar, desse modo, o limiar de detecção. Nos experimentos com somação de resposta de supra-limiar, ao invés de se utilizar somação de resposta com níveis de 
contraste sub-limiar para mensurar o máximo da função, utiliza-se somação de resposta de supra-limiar de contraste, onde a freqüência de teste é somada pixel a pixel às freqüências de fundo que são apresentadas a alto contraste. Nesse, enquanto o contraste da freqüência de teste é variado de acordo com a sensibilidade do voluntário, o contraste da freqüência de fundo é fixo acima do limiar (isto é, supralimiar). Desta forma, se um estímulo contendo apenas uma das freqüências de fundo é comparada a um estímulo em que a mesma freqüência de fundo é somada a freqüência de teste, a única forma de diferenciar entre os dois estímulos é detectar a presença da freqüência de teste em um destes. Informações mais detalhadas sobre este paradigma podem ser encontrado em Santos (1996, 1999) e Simas e colaboradores (Simas \& Dodwell, 1990; Simas \& Santos, 1998; Simas \& cols., 1992).

Durante o processo de filtragem, três tipos de respostas podem ocorrer: 1) aquelas em que determinadas freqüências de fundo podem se somar à freqüência de teste do filtro facilitando a sua detecção (efeito de somação); 2) aquelas em que as freqüências de fundo e de teste podem não se somar, não facilitando nem dificultando sua detecção (efeito independente); ou, 3) aquelas em que as freqüências de fundo podem inibir a percepção da freqüência de teste do filtro, dificultando a sua detecção (efeito inibitório). A sensibilidade ao contraste da freqüência de teste em função das freqüências de fundo, usando como referência o limiar absoluto de detecção da freqüência de teste, indicará a independência dos mecanismos de detecção ou os efeitos de somação ou inibição.

A interpretação dada ao paradigma de supra-limiar é: 1) se a superposição da freqüência de teste do filtro à freqüência de fundo facilita ou diminui o valor de contraste absoluto necessário à detecção, isto pode ser porque a freqüência de teste e a freqüência de fundo estão sendo processadas pelo mesmo mecanismo ou canal; 2) se a superposição da freqüência de teste à freqüência de fundo dificulta ou aumenta o valor de contraste absoluto, isto podem ser porque elas são processadas por canais distintos que interagem entre si possivelmente de forma antagônica; 3) se a superposição da freqüência de teste à freqüência de fundo não facilita e nem dificulta o valor de contraste absoluto (efeito independente), a freqüência de teste e a freqüência de fundo possivelmente estão sendo processadas por canais distintos que não interagem entre si.

A largura de banda de um filtro se refere à função de resposta para as freqüências espaciais desse filtro. Dentro desse contexto, espera-se que a curva de resposta para um filtro transmita informações 1) sobre a banda (ou bandas) de passagem do filtro; 2) sobre as freqüências em que o filtro responde menos ou não responde (deixa passar alguma coisa ou não deixa passar nada); e, 3) sobre aquelas freqüências que estão fora da faixa de resposta do filtro. É importante destacar que, quanto mais estreita a largura da banda, mais alta sua seletividade à freqüência espacial e vice-versa.

\section{O Presente Estudo}

Este estudo dá continuidade aos experimentos que procuram caracterizar a resposta do SVH para padrões de freqüências espaciais em coordenadas polares. Boa parte destes experimentos acreditam na possibilidade das áreas superiores do sistema visual (Ex.: área visual V4 e córtex infero-temporal, IT) processarem informação visual de forma em termos de freqüências radiais e angulares, o que pode envolver filtragem de faixas ou áreas inteiras do campo visual.

O presente estudo procura caracterizar mecanismos ou canais sintonizados para freqüências radiais moduladas pelo perfil cilíndrico de Bessel, $\mathrm{J}_{0}$. Até então, existem na literatura estimativas para filtros de freqüência angular de banda estreita (Santos, 1999; Simas, 1985; Simas \& Dodwell, 1990; Simas e cols., 1992; Simas \& Santos, 1998).

O objetivo principal foi estabelecer a existência de filtros de freqüências radiais de banda estreita utilizando padrões de freqüências espaciais modulados pela função cilíndrica de Bessel, isto é, estímulos elementares em coordenadas polares.

\section{Método}

\section{Participantes}

Participaram dos experimentos 5 voluntários de ambos os sexos com acuidade visual normal ou corrigida na faixa etária de 20-25 anos.

\section{Equipamentos e Estímulos}

Foi utilizado o sistema desenvolvido no próprio laboratório, que compreende um monitor de vídeo SONY-BVM-1910, com entrada "RGBsync" entrelaçada e interfaciado a um microcomputador 486 de $30 \mathrm{MHz}$ através de um placa de aquisição Data-Translation DT2853. Um programa escrito em linguagem $C$ foi desenvolvido no próprio laboratório para executar os experimentos. Uma cadeira giratória foi fixada a $150 \mathrm{~cm}$ da tela do monitor de vídeo. Uma tábua cinza foi situada acima do monitor de vídeo para onde o voluntário, entre uma apresentação e outra, deveria fixar o olhar com o objetivo de diminuir os efeitos de pós-imagem. A luminância média foi de 2,0 fL ajustada por um fotômetro do tipo SPOT METTER, com precisão de um grau, ASAHI PENTAX. A luminância mínima foi de 1,8 fL e 
a máxima de 2,2fL. O ambiente do laboratório era cinza para melhor controle da luminância.

Os estímulos de freqüências radiais foram gerados em tons de cinza e apresentados em tempo real no monitor. Todos os estímulos também eram circulares com um diâmetro de 7,25 graus de ângulos visuais a 150 $\mathrm{cm}$ de distância da tela (Figura 1).

As estimativas foram feitas utilizando pares de estímulos, estímulo de teste e estímulo de fundo. $\mathrm{O}$ estímulo de teste era composto pela freqüência de teste do filtro (1 ou $4 \mathrm{cpg}$ ) somada a uma das freqüências de fundo, por exemplo: 0,$2 ; 0,3 ; 0,5 ; 0,8 ; 1 ; 2 ; 3 ; 4 ; 5 ; 6$ ou 9 cpg. O estímulo de fundo foi a própria freqüência de fundo sozinha. A Figura 1 ilustra exemplos de pares de estímulos de freqüências radiais utilizados, por exemplo, para mensurar o filtro radial de $1 \mathrm{cpg}$.

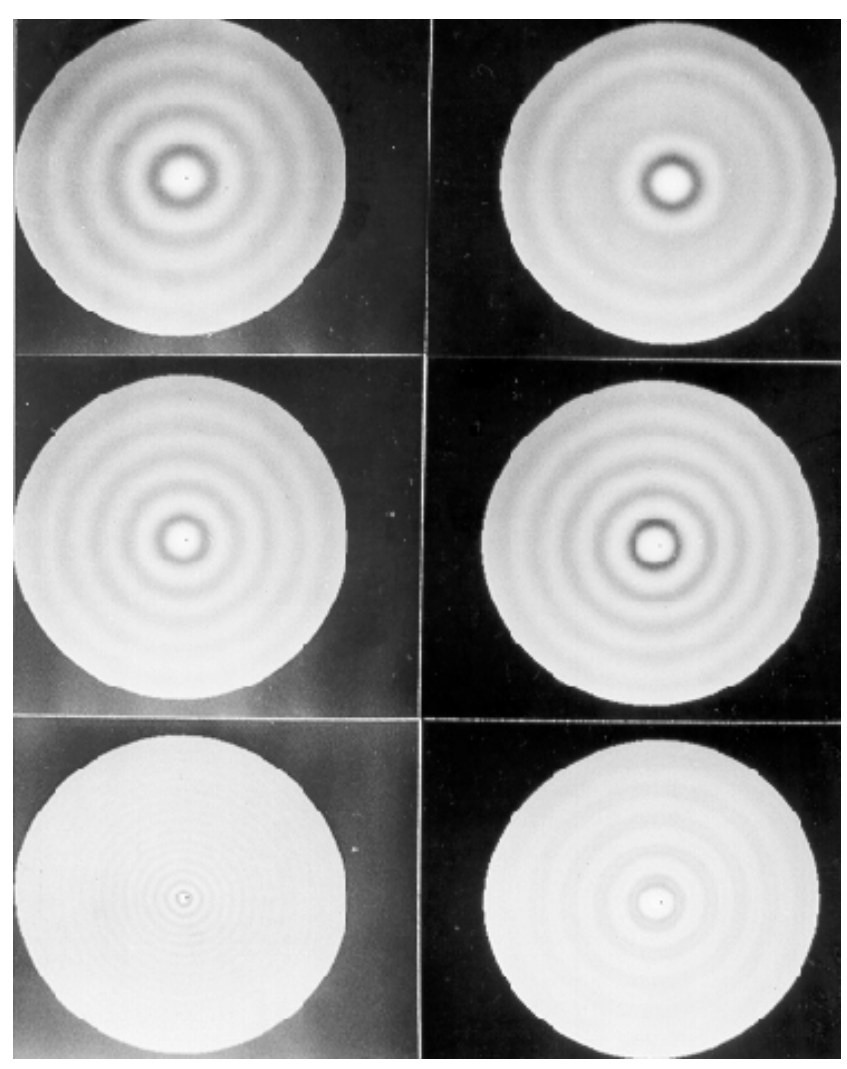

Figura 1. Exemplos de pares de estímulos de freqüências radiais utilizados para estimar o filtro de $1 \mathrm{cpg}$. Acima à esquerda EF de 0,8 cpg e á direita ET de 0,8+1 cpg, ao centro à esquerda EF de $1 \mathrm{cpg}$ e à direita ET de $1+1 \mathrm{cpg}$ e embaixo à esquerda $\mathrm{EF}$ de $3 \mathrm{cpg}$ e à direita ET de 3+1 $\operatorname{cpg}(\mathrm{EF}=$ Estímulo de fundo e ET = estímulo de teste $)$. Estímulos originalmente calibrados para serem vistos a $150 \mathrm{~cm}$ de distância antes de fotografados.

\section{Procedimento}

O procedimento de resposta de somação de supralimiar para cada filtro consistiu na apresentação sucessiva simples do par de estímulos e o voluntário teria que escolher dentre os dois estímulos qual destes continha a freqüência radial de teste do filtro somada à freqüência radial de fundo. $\mathrm{O}$ contraste da freqüência de fundo foi mantido constante e correspondia a cinco vezes o limiar absoluto de detecção da freqüência de teste do filtro de 1 ou $4 \mathrm{cpg}$, tanto no estímulo de teste como no estímulo de fundo. Somente o contraste supra-limiar da freqüência de teste era variada, diminuindo ou aumentando de acordo com o método da escolha forçada (Wetherill \& Levitt, 1965). O critério adotado para variar o contraste da freqüência de teste era o de três acertos consecutivos para decrescer uma unidade e um erro para acrescer da mesma unidade $(0,08 \%)$. Este procedimento experimental se baseia no cálculo da probabilidade de acertos consecutivos por parte do voluntário, ou seja, em cerca de 100-150 apresentações de escolhas entre os dois estímulos, o estímulo de teste é percebido $79 \%$ das vezes pelo voluntário.

A seqüência temporal foi iniciada com um sinal sonoro seguido imediatamente pela apresentação do primeiro estímulo por $2 \mathrm{~s}$, seguido de um intervalo entre estímulos de $1 \mathrm{~s}$, seguido pela apresentação do segundo estímulo por $2 \mathrm{~s}$ e da resposta do voluntário. A ordem de apresentação dos estímulos era aleatória. Se a resposta do voluntário fosse correta, ela era seguida por outro sinal sonoro e um intervalo de $3 \mathrm{~s}$ para a seqüência temporal se repetir. Em outras palavras, $3 \mathrm{~s}$ era o intervalo entre tentativas independente da resposta (ou escolha) ser correta ou não. O sinal sonoro que indica o início da apresentação do par de estímulos e o que indicava a escolha correta eram diferentes e discretos. A sessão experimental variava em duração dependendo dos erros e acertos do voluntário até proporcionarem um total de 10 máximos e 10 mínimos conforme requerido para o final automático da mesma. Sua duração era da ordem de 10-20 min.

Os voluntários foram orientados antes da sessão a pressionar a barra de espaço quando julgassem que o estímulo de teste tivesse sido apresentado primeiro e qualquer tecla acima da barra de espaço quando julgasse que o mesmo tivesse sido apresentado em segundo lugar, isto é, após o estímulo de fundo.

A ordem de mensuramento das freqüências para cada curva foi aleatorizada por sorteio pelo voluntário antes de mensurar cada uma das três curvas para cada um dos dois filtros. Todas as estimativas foram feitas à distância de 150 $\mathrm{cm}$, com visão binocular. 
Cada uma das 11 sessões experimentais requeridas para medir cada um dos dois filtros foi realizada pelo menos três vezes, em dias diferentes, por cada um dos 5 voluntários. Portanto, um total de 15 curvas (ou funções) foi medida para cada filtro gerando uma amostra de 300 valores para cada um dos 11 pontos estimados (cada freqüência de fundo correspondeu a um ponto e a uma sessão experimental).

\section{Resultados}

A Figura 2 mostra as curvas de respostas ao contraste para os filtros de freqüências radiais de 1 e 4 cpg. As freqüências radiais de fundo são apresentadas nos gráficos em função da quantidade de contraste necessário para identificar a presença da freqüência radial de teste no par que continha a soma. Estamos chamando este dado de limiar para a freqüência de teste somada a cada freqüência de fundo de limiar de contraste para o estímulo teste na soma. A linha horizontal representa a linha de base, que eqüivale ao limiar absoluto para detectar a freqüência de teste do filtro (1 ou $4 \mathrm{cpg}$ ) isolada.

Nosso tratamento estatístico foi estimar o erro padrão da média para cada distribuição de 300 valores mensurados para cada ponto e corrigidos para o tamanho da amostra pelo estatístico t-student para obter o nível de confiança de $99 \%$. Em experimentos anteriores, foi estabelecido que o erro padrão da média, corrigido pelo tamanho da amostra representando intervalo de confiança de $99 \%$ pelo estatístico $t$-student, de acordo com o número de valores mensurados, é um critério mais estrito do que a utilização da ANOVA ou teste $t$ para amostras correlacionadas. Por exemplo, quando os intervalos dos erros-padrão da média assim corrigidos se superpõem até mesmo pela metade, ainda assim um teste $t$-student para amostras correlacionadas revela diferenças entre as médias que são significativas $\operatorname{com} p<0,05$. Nos casos em que os erros barras não se sobrepõem, as médias são significativas com $p<0,001$. A ANOVA tende a mostrar interações e efeitos significativos em todos fatores e não acrescenta muita informação.

Os filtros de freqüências radiais de 1 e $4 \mathrm{cpg}$ se comportam de maneira semelhante, isto é, eles apresentam efeito de somação máxima em suas respectivas freqüências radiais de teste (1 e $4 \mathrm{cpg}$ ) circundadas de inibição nas freqüências vizinhas às freqüências radiais de teste (Figura 2).

As razões entre somação e inibição máximas foram da ordem de 2,18 e 2,0 para os filtros de 1 e 4 cpg, respectivamente. Estas razões foram encontradas nas freqüências vizinhas (por exemplo, $0,8 \mathrm{cpg}$ no caso do filtro radial de $1 \mathrm{cpg}$ e 3,0 cpg no caso do filtro radial de $4 \mathrm{cpg}$ ),

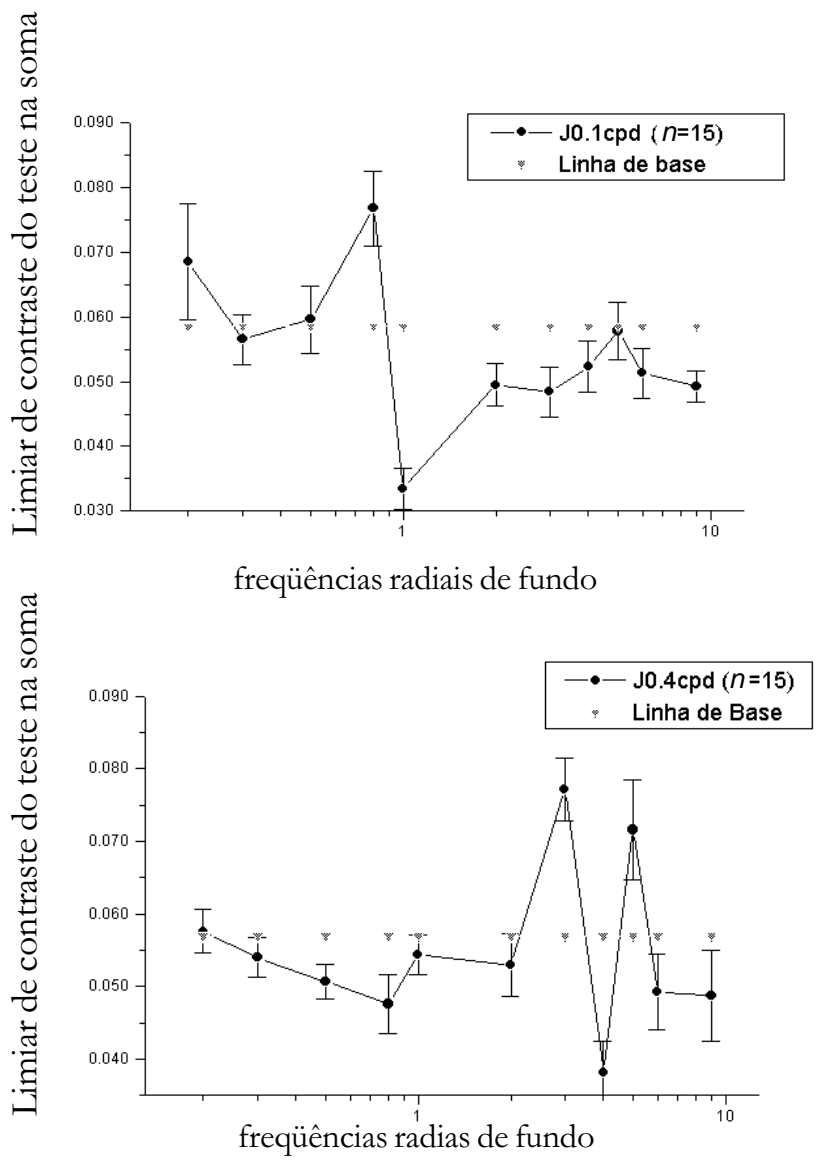

Figura 2. Mostra as curvas de resposta ao contraste da freqüência radial de teste do filtro de $1 \mathrm{cpg}$ acima e $4 \mathrm{cpg}$ abaixo somada às respectivas freqüências radiais de fundo representadas na abscissa.

indicando claramente efeitos de somação ou filtragem. Em outras palavras, a banda de passagem de cada filtro radial é circundada em ambos os lados por efeito de inibição.

Estes resultados são consistentes com a existência de filtragem de freqüências espaciais radiais de banda estreita na faixa de freqüência estudada.

\section{Discussão}

Teorias tradicionais em percepção visual da forma geralmente acreditam que o processamento visual é servido por canais ou filtros de banda-estreita que respondem seletivamente para freqüências e orientações espaciais (Manahilov \& Simpson, 2001). A noção de canal ou filtro supõe que um estímulo apresentado no campo visual não estimula todos os neurônios corticais que recebem informações daquela área da retina, mas apenas um grupo de neurônios seletivos para uma dimensão do 
estímulo, por exemplo, freqüência espacial. Assim, se um segundo estímulo que difere do primeiro ao longo da faixa de freqüência (ou uma outra dimensão qualquer) é apresentado na mesma área, ele ativará um grupo de neurônios diferente do primeiro. Em outras palavras, estímulos diferentes são processados por grupos ou subunidades de neurônios diferentes, os quais formam a base dos canais ou filtros.

A filtragem espacial realizada pelo SVH parece ter uma função crucial, pois permite ao sistema lidar seletivamente com uma diversidade muito grande de características simples e complexas de objetos em estágios anteriores que possivelmente são integradas em estágios posteriores, permitindo a reconstrução e interpretação do objeto ou da cena visual, que possivelmente resulta naquilo que denominamos de percepção visual da forma. A grande maioria de nossas idéias sobre o mundo é baseada na visão.

O objetivo principal do sistema visual talvez seja representar o ambiente visual da maneira mais concreta para a realidade na qual o sistema foi adaptado. Há quem acredite, que o sistema visual exista para derivar da imagem a informação que nós precisamos e não simplesmente para recriar a imagem projetada na retina (Braddick, Campbell \& Atkinson, 1978).

A existência de filtros de freqüência espacial de banda estreita no SVH foi demonstrado inicialmente com estudos psicofísicos para estímulos cartesianos verticais, grade senoidal, (Blakemore \& Campbell, 1969b; Graham, 1972; Sachs \& cols., 1971). Esses trabalhos iniciais indicaram que os canais que processavam banda estreita eram independentes. Entretanto, com o passar dos anos, novos estudos foram surgindo e verificou-se que esses canais podiam interagir entre si (Braddick \& cols., 1978; De Valois, 1977; Pollen \& Ronner, 1982; Tolhurst, 1972; Tolhurst \& Barfield, 1978). Em outras palavras, um modelo de canais múltiplos não implica necessariamente que em qualquer condição de estimulação e teste o sistema visual se comporte como completamente composto por canais de freqüências espaciais fortemente independentes (Sekuler, 1974).

Os nossos resultados com filtros de freqüência radial cilíndrica $\left(\mathrm{J}_{0}\right)$ de 1 e $4 \mathrm{cpg}$, também, demostram efeitos de somação secundários para freqüências radiais de fundo mais baixas e mais altas. Por exemplo, o filtro radial de 1 cpg demonstra efeito de somação relativo nas freqüências de fundo mais altas (2, 3, 4, 8 e 9 cpg), enquanto o filtro radial de $4 \mathrm{cpg}$ mostra somação relativa em freqüências radiais de fundo mais baixas $(0,8 \mathrm{cpg}) \mathrm{e}$ mais altas $(6$ e $9 \mathrm{cpg})$. Estes resultados mostram evidências para interações entre canais de freqüências espaciais em coordenadas polares. Resultados nesta direção já foram descritos na literatura para grade senoidal em coordenadas cartesianas (De Valois, 1977; Tolhurst, 1972; Tolhurst \& Barfield, 1978) e também para estímulos de freqüências angulares em coordenadas polares (Simas, 1985; Simas \& Dodwell, 1990; Simas \& cols., 1992). Por exemplo, alguns trabalhos com grade senoidal encontraram casos de sensibilidade aumentada para freqüências espaciais a cerca de uma oitava da freqüência da grade de teste (De Valois, 1977; Tolhurst, 1972; Tolhurst \& Barfield, 1978). Esses autores discutiram que isso constitui evidência para interações entre canais de freqüências espaciais. Alguns resultados também mostram interações entre o fundamental e o terceiro harmônico (Pollen \& Ronner, 1982). Apesar de estes resultados serem com padrões cartesianos ao invés de polares, são interessantes na medida em que os nossos resultados para filtros radiais em coordenadas polares também apresentam interação entre canais de freqüência espacial radial de banda estreita. Estes resultados também demostram que os canais para freqüências espaciais radiais não são totalmente independentes.

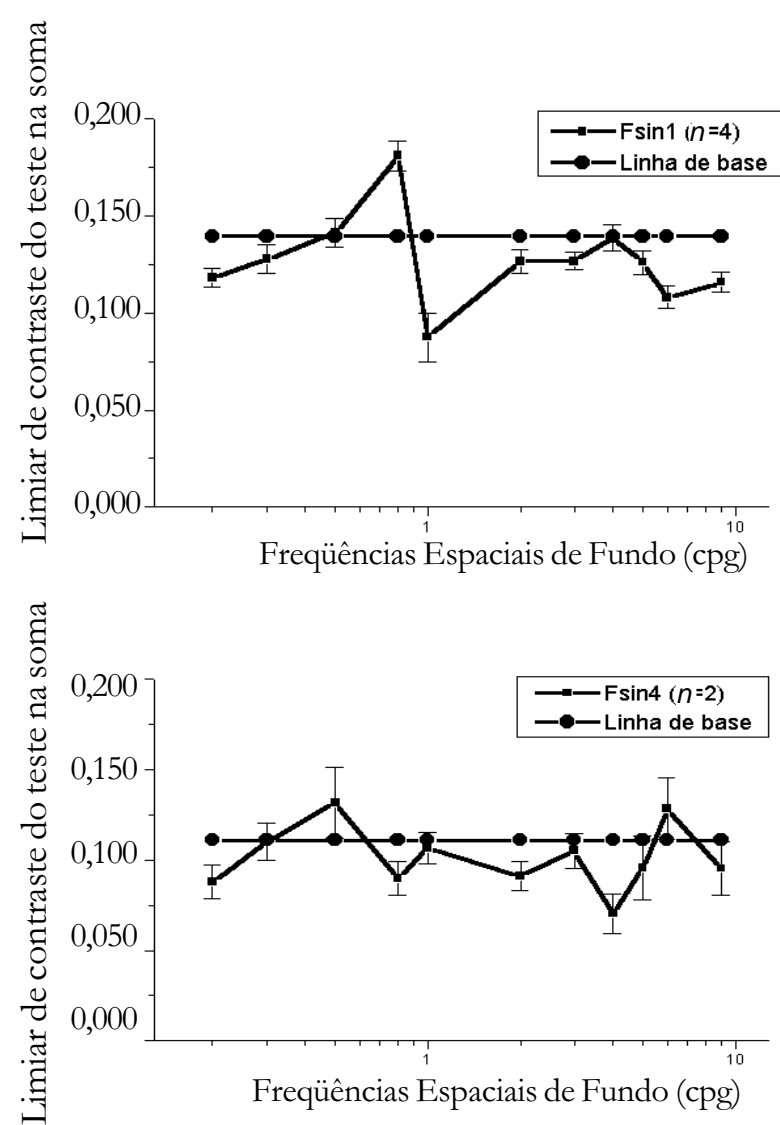

Figura 3. Mostra as curvas de resposta ao contraste para freqüência espacial (grade senoidal) para os filtros de 1 cpg acima e $4 \mathrm{cpg}$ abaixo somada às respectivas freqüências espaciais de fundo representadas na abscissa. 
A Figura 3 mostra as curvas de resposta ao contraste para filtros de freqüência espacial (grade senoidal) de $1 \mathrm{e}$ 4 cpg mensuradas em nosso laboratório com o mesmo método psicofísico utilizado para mensurar os filtros de freqüência radial de 1 e $4 \mathrm{cpg}$. Apesar destas medidas terem sido feitas com equipamentos diferentes, as curvas de filtragem para freqüência radial e grade senoidal se comportam de maneira semelhante, ou seja, ambas mostram somação máxima na freqüência de teste dos filtros de 1 e 4 cpg (Figura 3). As curvas de filtragem para padrões radiais e grades senoidais deixam claras a seletividade do SVH para esses dois tipos de padrões espaciais com características espaciais um tanto diferente. Isto é, grade senoidal é um estímulo clássico cuja luminância vária senoidalmente e unidirecionamente no espaço e pode ser facilmente definido em um sistema de coordenadas cartesianas. Ao passo que estímulo radial modulado pela função cilíndrica de Bessel é um estímulo cuja luminância varia senoidalmente e unidirecionalmente na direção radial (ou seja, a luminância varia do centro para a periferia desse tipo de padrão), o que o torna um estímulo facilmente definido em um sistema de coordenas polares com a origem do sistema de coordenadas no centro do estímulo. Exemplos de estímulos de freqüência radial, freqüência angular e freqüência radial/angular acoplada pode ser encontrado em Santos $(1996,1999)$ e Santos e Simas (2001b). Esses trabalhos também apresentam funções matemáticas utilizadas para gerar estímulos dessa natureza.

A Figura 4 ilustra exemplos de pares de estímulos de freqüências espaciais, grade senoidal, utilizados para estimar, por exemplo, o filtro espacial de $1 \mathrm{cpg}$.

Os resultados encontrados para os filtros radiais de 1 e 4 cpg com o paradigma de somação de resposta de supra-limiar, também, são semelhantes aos resultados encontrados por Simas e colaborados (Simas, 1985; Simas \& Dodwell, 1990; Simas \& cols., 1992) para freqüências angulares, em coordenadas polares, com o mesmo método psicofísico. Ou seja, Simas e colaboradores também encontram filtragem ou somação máxima e relativa nas freqüências angulares de teste dos filtros estudados circundadas por inibições.

Talvez o pressuposto básico aqui seja a idéia de que, dependendo do filtro espacial (grade senoidal, radial ou angular), mecanismos diferentes interdependentes e intradependentes sejam ativados (efeito de inibição, somação e independência dos canais para freqüências espaciais radiais). Os filtros investigados aqui podem não ser tão perfeitos quanto o filtro ideal (que deixa a quantidade desejada inalterada e reduz todos os outros valores a zero), porém fica a idéia que o sistema de

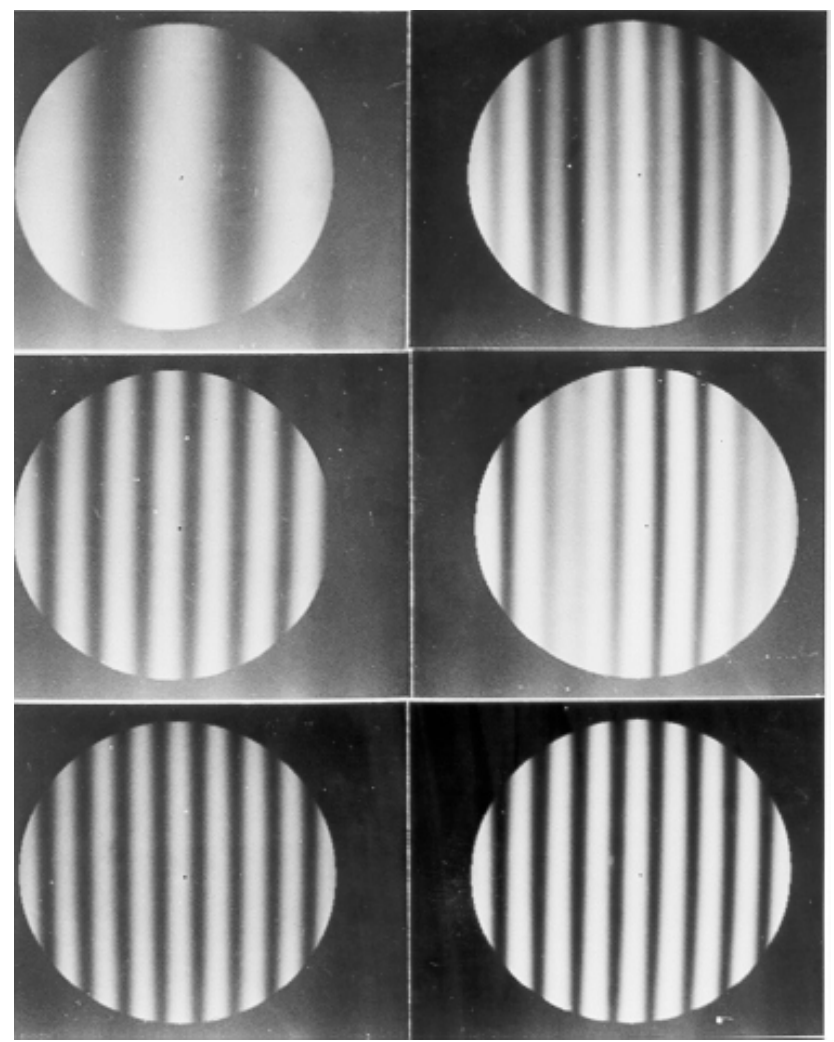

Figura 4. Exemplos de pares de estímulos de freqüências espaciais, grade senoidal, utilizados para estimar o filtro espacial de 1 cpg. Acima à esquerda EF de 0,3 cpg e á direita ET de 0,3+1 cpg, ao centro à esquerda EF de 0,8 cpg e à direita ET de 0,8+1 cpg e embaixo à esquerda $\mathrm{EF}$ de $1 \mathrm{cpg}$ e à direita ET de 1+1 cpg (EF = Estímulo de fundo e ET = estímulo de teste). Estímulos originalmente calibrados para serem vistos a $150 \mathrm{~cm}$ de distância antes de fotografados.

filtragem executado pelo SVH é muito robusto, o que nos leva a crer que a circuitaria visual dispõe de mecanismo para se ajustar a qualquer tipo de padrão de freqüências espaciais em coordenadas polares ou cartesianas. Desta forma, o sistema visual extrai as características necessárias para reconhecer os detalhes que diferenciam um padrão de outro de acordo com as "suas necessidades".

Em síntese, estes resultados fornecem evidências para a existência de filtros distintos sintonizados para percepção de padrões simétricos em coordenadas polares. Entretanto, por outro lado, estes resultados sobre filtragem radial não podem provar por si só que o sistema visual utiliza este tipo de filtro para processar e reconhecer objetos ou uma cena visual. Portanto, o fato de o SVH ser sensível a estímulos desta natureza, por si só é um indício forte da importância destes estímulos no processamento visual de forma. 


\section{Evidências neurofisiológicas para filtragem de estímulos espaciais em coordenadas polares:}

Existem fortes evidências, baseadas em estudos psicofísicos e neurofisiológicos, de que o processamento linear ou filtragem espacial local sintonizada para orientação e freqüência espacial de estímulos projetados na retina ocorre nos estágios iniciais da visão, isto é, em neurônios do córtex visual primário, V1 (De Valois \& De Valois, 1988; Manahilov \& Simpson, 2001; Wilkinson \& cols., 2000; Wilson \& Wilkinson, 1998; Wilson \& cols., 1997). Ao contrário, até o momento não existe nenhum relato que unidades neurais da área visual primária, V1, executem filtragem de estímulos em coordenadas polares, amostrando áreas grandes do campo visual. Por outro lado, existem dados baseados em estudos psicofísicos em humanos (Wilkinson \& cols., 1998; Wilson \& Wilkinson, 1997, 1998; Wilson \& cols, 1997), neurofisiológicos em primatas (Bruce, Desimone \& Gross, 1981; Desimone, 1991; Desimone \& Schein, 1987; Gallant \& cols., 1993, 1996; Hegde \& Van Essen, 2000; Heywood, Gadotti \& Cowey, 1992, Merigan, 1996; van Essen, Anderson \& Felleman 1992; Young, 1992) e imagem de ressonância magnética funcional em humanos (Wilkinson \& cols., 2000), de que o processamento da informação que ocorre em vias intermediárias (Ex.: área visual V4) e áreas mais avançadas (Ex.: córtex visual inferotemporal, IT) do sistema visual são modeladas por filtros espaciais globais em coordenadas polares. Alguns desses trabalhos citados acima destacam que a área V4 pode formar o principal estágio intermediário da visão de forma de V1 para IT (Heywood \& cols., 1992, Merigan, 1996; van Essen \& cols., 1992; Wilkinson \& cols., 2000; Young, 1992). Assim, é possível que a filtragem e integração de padrões em coordenadas polares ocorram em áreas visuais extra-estriado (Ex.: V4 e IT). O estudo de Wilkinson e colaboradores (2000) com a técnica de imagem de ressonância magnética funcional em humanos reforça os estudos psicofísicos com humanos e neurofisiológicos com primatas que demonstraram inicialmente que estímulos em coordenadas polares são processados em áreas extra-estriado (Ex.: V4 e IT). Além disto, este estudo reforça a idéia de que o sistema visual de humanos e primatas são muito semelhantes. Inclusive, em artigo recente, Connor (2000) afirma que macacos percebem objetos de maneira semelhante a humanos.

A idéia geral que fica, baseado nos nossos dados e nos dados encontrados na literatura, é que se não existem mecanismos distintos seletivos a freqüências angulares, radiais, radiais/angulares acopladas e grade senoidal, pelo menos devem existir mecanismos distintos ou áreas distintas para estímulos em coordenadas polares e para estímulos em coordenadas cartesianas.

Todos os resultados apresentados e discutidos neste trabalho são consistentes com a possibilidade de que estímulos em coordenadas polares são importantes para caracterizar a resposta do SVH.

\section{Referências}

Amidor, I. (1997). Fourier spectrum of radially periodic images. Journal of the Optical Society of America, 14, 816-826.

Blakemore, C. \& Campbell, F. C. (1969a). Adaptation to spatial stimuli. Journal of Physiology, 200, 11-12.

Blakemore, C. \& Campbell, F. C. (1969b). On the existence of neurons in the human visual system selectively sensitive to the orientation and size of retinal images. Journal of Physiology, 203, 237-260.

Blakemore, C., Nachmias, J. \& Sutton, P. (1970). The perceived spatial frequency selective neurones in the human brain. Journal of Physiology, 210, 727-750.

Braddick, O., Campbell, F. W. \& Atkinson, J. (1978). Channels in vision: Basic aspects. Em R. Held, H. W. Leibowitz \& H. L. Teuber (Orgs.), Handbook of sensory physiology: Perception (Vol. V, pp. 3-38). New York: Springer-Verlag.

Bruce, C. J., Desimone, R. \& Gross, C. G. (1981). Visual properties of neurons in a polysensory area in superior temporal sulcus of the macaque. Journal of Neurophysiology, 46, 369-384.

Campbell, F. W. \& Maffei, L. (1970). Electrophysiological evidence for the existence of orientation and size detectors in the human visual system. Journal of Physiology, 207, 635-652.

Campbell, F. W. \& Maffei, L. (1974). Contrast and spatial frequency. Scientific American, 231, 106-114.

Campbell, F. W. \& Robson, F. G. (1968). Application of the Fourier analysis to the visibility of gratings. Journal of Physiology, 197, 551-566.

Connor, C. E. (2000). Visual perception: Monkeys see things our way. Current Biology, 10,836-838.

De Valois, K. (1977). Spatial frequency adaptation can enhance contrast sensitivity. Vision Research, 17, 1057-1065.

De Valois, R. L. \& De Valois, K. K. (1988). Spatial vision. New York: Oxford University Press.

Desimone, R. (1991). Face-selective cells in the temporal cortex of monkeys. Journal of Cognitive Neuroscience, 3, 1-8.

Desimone, R. \& Schein, S. J. (1987). Visual properties of neurons in area V4 of macaque: Sensitivity to stimulus form. Journal of Neurophysiology, $57,835-867$

Gallant, J. L., Brau, J. \& van Essen, D. C. (1993). Selectivity for polar hyperbolic, and cartesian gratings in macaque visual cortex. Science, 259, 100103.

Gallant, J. L., Connor, C. E., Rakshit, S., Lewis, J. W. \& van Essen, D. C. (1996). Neural responses to polar, hyperbolic, and cartesian gratings in area V4 of the macaque monkey. Journal of Neurophysiology, 76, 27182739.

Graham, N. (1972). Spatial frequency channels in the human visual system: Effects of luminance and pattern drift rate. Vision Research, 12, 53-63.

Hegde, J. \& Van Essen, D. C. (2000). Selectivity for complex shapes in primate visual area V2. Journal of Neuroscience, 20, RC61.

Hess, R. F., Wang, Y.-Z., Demanins, R., Wilkinson, F. \& Wilson, H. R. (1999). A deficit in strabismic amblyopia for global shape detection. Vision Research, 39, 901-914.

Heywood, C. A., Gadotti, A. \& Cowey, A. (1992). Cortical area V4 and its role in the perception of color. Journal of Neuroscience, 12, 4056-4065.

Kelly, D. H. (1960). Jo stimulus patterns for vision research. Journal of the Optical Society of America, 50, 1115-1116. 
Kelly, D. H. (1982). Motion and vision: IV. Isotropic and anisotropic spatial response. Journal of the Optical Society of America, 72, 432-439.

Kelly, D. H. \& Magnuski, H. S. (1975). Pattern detection and the two demensional Fourier transform: Circular targets. Vision Research, 15, 911-915.

Kulikowski, J. J. \& King-Smith, P. E. (1973). Spatial arrangement of line, edge and grating detectors relealed by sub-threshold summation. Vision Research, 13, 1455-1478.

Maffei, L. \& Fiorentini, A. (1973). The visual cortex as a spatial frequency analyzer. Vision Research, 13, 1255-1267.

Manahilov, V. \& Simpson, W. A. (2001). Energy model for contrast detection: Spatial-frequency and orientation selectivity in grating summation. Vision Research, 41, 1547-1560.

Merigan, W. H. (1996). Basic visual capabilities and shape discrimination after lesions of extrastriate area V4 in macaques. Visual Neuroscience, 13, 51-60.

Pantle, A. \& Sekuler, R. (1968). Size detecting mechanisms in human vision. Science, 162, 1146-1148.

Pollen, D. A. \& Ronner, S. F. (1982). Spatial computation performed by simple and complex cells in the visual cortex of the cat. Vision Research, 22, 101-118.

Sachs, M. B., Nachmias, J. \& Robson, J. G. (1971). Spatial frequency channels in human vision. Journal of the Optical Society of America, 61, 1176-1186.

Santos, N. A. (1996). Sistema visual bumano: Filtragem de freqüencias radiais moduladas por perfis de Bessel j0, j1, j2, j4, j8 e j16. Dissertação de Mestrado nãopublicada, Curso de Pós-Graduação em Neurociências e Comportamento, Instituto de Psicologia, Universidade de São Paulo. São Paulo, S. P.

Santos, N. A. (1999). Sistema visual bumano: Curvas de sensibilidade e filtragem de frequiencias angulares, radiais e radial/ angulares acopladas. Tese de Doutorado não-publicada, Curso de Pós-Graduação em Neurociências e Comportamento, Instituto de Psicologia, Universidade de São Paulo. São Paulo, S. P.

Santos, N. A. \& Simas, M. L. B. (2001a). Percepção e processamento visual da forma: Discutindo modelos teóricos atuais. Psicologia: Reflexão e Crítica, 14, 151-160.

Santos, N. A. \& Simas, M. L. B. (2001b). Função de sensibilidade ao contraste: Indicador da percepção visual da forma e da resolução espacial. Psicologia: Reflexão e Crítica, 14, 589-597.

Sekuler, R. (1974). Spatial vision. Annual Review of Psychology, 25, 195-232.

Simas, M. L. B. (1985). Linearity and domain invariance in the visual system. Tese de Doutorado, não-publicada, Queen's University at Kingston, Ontario, Canada, 1985. University Microfilms International, 86, 17940.

Simas, M. L. B. \& Dodwell, P. C. (1990). Angular frequency filtering: A basis for pattern decomposition. Spatial Vision, 5, 59-74.

Simas, M. L. B., Frutuoso, J. T. \& Vieira, F. M. (1992). Inhibitory sidebands in multiple angular filters in the human visual system. Brazilian Journal of Medical and Biological Research, 25, 919-923.
Simas, M. L. B. \& Santos, N. A. (1997). Human visual contrast detection of radial frequency stimuli defined by Bessel profiles $j 0, j 1, j 2, j 4, j 8$ and j16 and its relation to angular frequency [Resumos]. Em IEEE Computer Science (Org.), Anais do II Workshop on Cybernetic Vision (pp. 219-224). São Carlos, S. P.: IEEE Computer Science.

Simas, M. L. B. \& Santos, N. A. (1998). Human frequency response functions of harmonic 2, 4, 8, and 16 cycle angular frequency filters [Resumos]. Em IEEE Computer Science (Org.), Anais do Internacional Symposium on Computer Graphics, Image Processing and Vision (pp. 312-319). Rio de Janeiro: IMPA.

Tolhurst, D. J. (1972). Adaptation to square-wave gratings: Inhibition between spatial frequency channels in the human visual system. Journal of Physiology, 226, 231-248.

Tolhurst, D. J. \& Barfield, L. P. (1978). Interactions between spatial frequency channels. Vision Research, 18, 851-858.

van Essen, D. C., Anderson, C. H. \& Felleman, D. J. (1992). Information processing in the primate visual system: An integrated systems perspective. Science, 255, 419-423.

Verrall, S. C. \& Kakarala, R. (1998). Disk-harmonic coefficients for invariant pattern recognition. Journal of the Optical Society of America 15, 389-401.

Wetherill, G. B. \& Levitt, H. (1965). Sequential estimation of points on a psychometric function. The British Journal of Mathematical and Statistical Psychology, 48, 1-10.

Wilkinson, F., Wilson, H. R. \& Habak, C. (1998). Detection and recognition of radial frequency patterns. Vision Research, 38, 3555-3568.

Wilkinson, F., James, T. W., Wilson, H. R., Gati, J. S., Menon, E. S. \& Goodale, M. A. (2000). An MRI study of the selective activation of human extrastriate form vision areas by radial and concentric gratings. Current Biology, 10, 1455-1458.

Wilson, H. R. \& Wilkinson, F. (1997). Evolving concepts of spatial channels in vision: From independence to nonlinear interactions. Perception, 26, 939-960.

Wilson, H. R. \& Wilkinson, F. (1998). Detection of global structure in glass patterns: Implications for form vision. Vision Research, 38, 29332947.

Wilson, R. W., Wilkinson, F. \& Asaad, W. (1997). Concentric orientation summation in human form vision. Vision Research, 37, 2325-2330.

Young, M. P. (1992). Objective analysis of topological organization of the primate cortical visual system. Nature, 358, 152-155.

Recebido: 17/10/2001

Revisado: 11/03/2002

Aceito: 27/03/2002

Sobre os autores

Natanael Antonio dos Santos é Doutor em Psicologia pelo Instituto de Psicologia da Universidade de São Paulo (2000), área de concentração Neurociências e Comportamento. Pesquisador do CNPq, Bolsa DCR, vinculado ao Departamento de Psicologia da UFPE.

Maria Lúcia de Bustamante Simas é Doutora em Psicologia pela Queen‘s University, Ontario, Canadá. Professora Adjunta do Departamento de Psicologia da UFPE. 


\section{NEPF}

Núcleo de Estudos em Psicologia Fenomenológica

Pelo avanço e difusão de instrumental metodológico de linhagem fenomenológica na pesquisa em psicologia.

Prof. William B. Gomes

Curso de Pós-Graduação em Psicologia

Instituto de Psicologia

Universidade Federal do Rio Grande do Sul

Rua Ramiro Barcelos 2600

90035.003 Porto Alegre -RS

E-mail: gomesw@.ufrgs.br

Visite nossa Homepage: 